\title{
1 Biogenic methane cycling is controlled by microbial cohorts
}

2 Rhiannon Mondav ${ }^{1,2}$, Gaëtan Martin $^{3}$, Sari Peura ${ }^{3 *}$, Sarahi L. Garcia ${ }^{2}$

3

4

51 Institute of Ecology and Genetics, Uppsala University, 75236 Uppsala, Sweden

62 Department of Ecology, Environment, and Plant Sciences, Science for Life Laboratory,

7 Stockholm University, 10691 Stockholm, Sweden

83 Department of Forest Mycology and Plant Pathology, Science for Life Laboratory, Swedish

9 University of Agricultural Sciences, 75007 Uppsala, Sweden

10 * Current address: Swedish Nuclear Fuel and Waste Management Co

14 Corresponding author: Sarahi L Garcia <sarahi.garcia@su.se>

17 Keywords: methane, carbon cycle, networks, metabolic prediction, community

19 Running title: Methane cycling model communities 


\section{Abstract}

22 The generation and consumption of methane by aquatic microbial communities is an

23 important contribution to the global carbon budget. We sought to broaden understanding of

24 consortia members and interactions by combining multiple methods including analysis of

25 natural and cultivated microbial communities. By analysing the microbial community

26 composition and co-occurrence patterns of a lake time-series we were able to identify

27 potential consortia involved in methane cycling. In combination with methane flux, we also

28 analysed the community composition and co-occurrence patterns of reduced microbial model

29 communities with inoculum from the same lake. While the network analyses confirmed many

30 known associations, when combined with results from mixed cultures, we noted new players

31 in methane cycling. Cultivated model communities were shown to be an effective method to

32 explore the rarer but still important players in methane cycling and for identifying new

33 putative members. Here we show that using multiple methods to approach the complex

34 problem of methane cycling consortia yields not just insights into the known taxa but

35 highlights potential new members creating new hypotheses to be tested. 


\section{Introduction}

39 Methane emissions from biogenic sources are determined by the ratio and activity of

40 methanogenic microbes producing and methanotrophic microbes consuming this powerful

41 greenhouse gas. Considering that methane is responsible for $17 \%$ to $20 \%$ of the radiative

42 forcing component of global warming $(1,2)$, elucidating all players in the methane cycle

43 involved in the net methane emissions from various biogenic sources is of utmost importance.

44 It is known that methanotrophs can oxidize between 30 and $99 \%$ of $\mathrm{CH} 4$ produced in the

45 sediments and the water column before it reaches the atmosphere (3-6). However, the

46 detection of methanotrophs in an environment does not necessarily correlate with

47 methanotrophic activity, and other factors may determine whether methane is oxidized (7).

48 One factor that may affect the rate or existence of methanotrophy is the composition of the

49 microbial community. For example, it has been shown that aerobic methanotrophs can be

50 active in anoxic waters when co-occurring with photosynthetic organisms (8), suggesting that

51 the methanotrophs utilize oxygen liberated by these phototrophs for methane oxidation.

52 However, discovering and pinpointing more of these symbiotic interactions is difficult in

53 natural environments as the number of possible interacting organisms is very high. Methods

54 to cultivate communities with reduced diversity have been developed (9) to overcome this

55 limitation and study organisms in a model communities in conditions mimicking their natural

56 habitat. In this method, environmental samples are diluted in filtered water from their own

57 environment and microorganisms subsampled for cultivation. These so-called model

58 communities are a powerful way to study the microbial interaction in a semi-natural

59 environment (10). Model communities also provide tools for building hypotheses that could

60 then be further tested by looking into interaction networks in time series of natural

61 environments (11).

62 For this study, we established 177 dilution-cultures model communities using samples from 
63 Lake Lomtjärnan, a lake in Sweden with high methane concentrations in the water column to

64 examine the methane cycling capacity of microbial assemblages. We also utilized a 2-week

65 depth discrete timeseries taken from March to April 2016 to examine the network around the

66 methane oxidizing communities in the actual lake. We hypothesize that the capacity to

67 produce or oxidize methane is not only related to the presence of organisms with the known

68 ability to produce or use methane, but that their cohorts might be key controllers in methane

69 cycling.

70

71 Materials and methods

72 Lake water collection and media preparation

73 Environmental samples for the time-depth-series were collected and published previously

74 (12). In brief, a sampling campaign including six time points was done in winter the last week

75 of March and the first week of April 2016 on Lake Lomtjärnan. This small forest lake had an

76 ice cover at the time of sampling and is located in central west region (Jämtland) of Sweden

77 (Fig 1A). The surface area of the lake is about 1 ha, and the maximum depth is $3.5 \mathrm{~m}$. The

78 lake is located on a mire surrounded by a coniferous forest. At each sampling occasion,

79 samples at six different depths $(0.65 \mathrm{~m}, 1.0 \mathrm{~m}, 1.35 \mathrm{~m}, 1.85 \mathrm{~m}, 2.35 \mathrm{~m}, 2.75 \mathrm{~m})$ were taken to

80 create a time-depth-series of the lake totalling 35 samples. The deepest depth was not taken

81 on the first sampling because samples were taken in a location where the max depth was

82 around $2.35 \mathrm{~m}$.

83 Water for the time-depth-series was collected using a depth-discrete Limnos tube-sampler

84 (Limnos, Poland) and the water was subsequently filtered through a Sterivex filter $(0.22 \mu \mathrm{m})$

85 and the filters were stored immediately in liquid nitrogen for later DNA extraction. Water to

86 be used for both growth media and inoculum was collected from the anoxic layer on the sixth

87 sampling occasion of the lake in April 2016 and again in September 2017. 
88 The water for media was collected using the same depth-discrete Limnos tube-sampler

89 (Limnos, Poland) and was quickly poured into 1 litre Schott bottles which were filled and let

90 to overflown to remove all the oxygenic water prior to closing the bottles. These bottles were

91 then kept in the dark at $4^{\circ} \mathrm{C}$ for 2 days to ensure anoxia. The water for media was filtered

92 twice through $0.2 \square \mu \mathrm{m}$ Sterivex filters (Millipore) inside the anaerobic glove box, and the

93 filtered-sterilized media was collected in a sterile Schott bottle and closed. These bottles were

94 further exposed to UV light for $10 \mathrm{~min}$.

95 The inoculum for the cultures was collected using the Limnos tube-sampler in a falcon tube

96 and was then flash frozen with liquid nitrogen and kept in $-80{ }^{\circ} \mathrm{C}$ until the cultures were

97 established. The following steps of the preparation were performed under anoxic atmosphere

98 using an anaerobic glove box.

$100 \quad$ Preparation of serum bottles, cell inoculant and cultures

$10120 \mathrm{ml}$ serum bottles, crimps, and stoppers were autoclaved at $120^{\circ} \mathrm{C}$ for $20 \mathrm{~min}$. To limit the 102 risk of potential bactericides such as benzyltoluenes and phenylalkanes, the stoppers were 103 autoclaved submerged in deionized water, and subsequently rinsed and boiled in sterile

104 deionized water and finally cooled in sterile deionized water. After autoclaving, the serum

105 bottles were sealed with these sterilised and detoxified butyl rubber stoppers and aluminium

106 crimps. The incubation vessels were assembled under a laminar flow, using autoclaved tools,

107 before being flushed at least 3 times with nitrogen to remove O2. To maintain the sterility of

108 the bottles during the flushing operation, new sterile needles were used for each bottle and the

109 stoppers were carefully disinfected with $90 \%$ ethanol. Furthermore a $0.2 \mu \mathrm{m}$ filter was used

110 to prevent contamination from the $\mathrm{N} 2$ gas flow.

111 The sealed sterile and anaerobic serum bottles were supplemented with $10 \mathrm{ml}$ of media, 112 a.k.a. filtered lake water. Subsamples of the unfiltered lake water from Lomtjärnan April 
1132016 (average 5x106 cells/ml) and September 2017 were run through a flow cytometer to

114 estimate cell concentration. Based on those values, another subsample, not exposed to the

115 atmosphere was diluted with lake-water-media to approximatively $50 \mathrm{cells} / \mathrm{ml}$. To inoculate

116 the bottles, $1 \mathrm{ml}$ of this 50 cells $/ \mathrm{ml}$ solution was then injected using sterile needles and

117 syringes into the $20 \mathrm{ml}$ serum bottles. The number of cultures prepared were: 98 from 2016

118 and 79 from 2017. A few bottles were incubated without inoculum as media control cultures.

119 To test for the potential for anoxic methane oxidation $4 \mathrm{ml}$ of a mix of $\mathrm{CH} 4$ and $\mathrm{CO} 2$ with a 120 ratio of $80 / 20 \%$ were injected into each bottle (Figure 1B).

121 Bottles were then incubated at $11^{\circ} \mathrm{C}$ for two months in the dark, to mimic lake conditions, for

122 the samples taken in the autumn and under dim light for the samples collected in the spring.

123 After the two-month incubation, $1 \mathrm{ml}$ of the headspaces was sampled for gas analysis.

124 Methane content was measured using the gas analyser Biogas 5000 (Geotechnical

125 Instruments, UK) and gas chromatography (Clarus 500, Perkin Elmer, USA, Polyimide

126 Uncoated capillary column $5 \mathrm{~m} \times 0.32 \mathrm{~mm}$, FID detector). At the same time as gas sampling

$127200 \mu \mathrm{l}$ of the cultures were collected and preserved at $-80^{\circ} \mathrm{C}$ for DNA analysis.

$16 S$ rRNA gene amplicon preparation and sequencing

130 DNA was amplified directly from $1 \mu$ of culture. Library preparation for 16S rRNA gene 131 analysis was done following a two-steps Polymerase Chain Reaction (PCR) protocol, as 132 described in Mondav et al 2020 (11). All PCRs were conducted in $20 \mu$ l of volume using 0.02

$133 \mathrm{U} / \mu$ l Phusion high fidelity DNA polymerase, $1 \mathrm{X}$ Q5 reaction buffer (NEB, UK), $0.25 \mu \mathrm{M}$ 134 primers and $200 \mu \mathrm{M}$ dNTP mix and $1 \mu \mathrm{l}$ mixed culture template. The first step was 135 performed in triplicate with primers 341F (3'-CCTACGGGNGGCWGCAG-5') and 805NR 136 (3'-GACTACNVGGGTATCTAA-5') (13). The thermal program consisted of 20 cycles with 137 an initial $98^{\circ} \mathrm{C}$ denaturation step for $10 \mathrm{~min}$, a cycling program of $98^{\circ} \mathrm{C}$ for 10 seconds, $48^{\circ} \mathrm{C}$ 
138 for 30 seconds, and $72^{\circ} \mathrm{C}$ for 30 seconds and a final elongation step at $72^{\circ} \mathrm{C}$ for 2 minutes.

139 Triplicate PCR reactions were then pooled and purified with magnetic beads (Sera-MagTM

140 Select, GE Healthcare, Chicago, United States of America), and $2 \mu$ l of the purified products

141 were used at a template for a second stage PCR, where indexed primers were added. The

142 second thermal program consisted of 15 cycles with an initial $98^{\circ} \mathrm{C}$ denaturation step for 30

143 seconds, a cycling program of $98^{\circ} \mathrm{C}$ for 10 seconds, $66^{\circ} \mathrm{C}$ for 30 seconds, and $72^{\circ} \mathrm{C}$ for 30

144 seconds and a final elongation step at $72^{\circ} \mathrm{C}$ for 2 minutes. Following amplification, PCR

145 products were again purified with magnetic beads and quantified with Qubit ${ }^{\mathrm{TM}}$ using the

146 Qubit $^{\mathrm{TM}}$ dsDNA HS Assay Kit (Invitrogen ${ }^{\mathrm{TM}}$ ). Finally, $15.6 \mu \mathrm{g}$ of each indexed and purified

147 PCR product were pooled before submission of the sample to the Science for Life Laboratory

148 SNP/SEQ sequencing facility hosted by Uppsala University (Uppsala, Sweden). Sequencing

149 was done using Illumina Miseq in paired-end mode with 300bp and v3 chemistry.

150

151 Amplicon bioinformatics

152 Sequence processing was performed with Mothur 1.41.0 following the MiSeq SOP (14), with

153 the exception that clustering to operational taxonomic units (OTUs) was done using

154 VSEARCH (15) as implemented in Mothur (16). Taxonomy was assigned against the Silva

155132 database (17). Amplicon sets were separated into groups of sample or inoculant origin:

156 time-depth-series lake communities, anaerobic lake cultures 2016, anaerobic lake cultures

157 2017, and 2 un-inoculated media controls. Nine lake-2017 cultures produced only a few

158 sequences $(<20)$ and were removed from the dataset. Remaining sequences were then

159 subsampled once using Qiimes (18) single rarefaction to an even depth of 600 reads per

160 sample for the un-inoculated media, and 2000 reads per sample for all others. This resulted in

161 final sample counts: 35 lake time-depth-series, 93 lake-2016 cultures and 70 lake-2017

162 cultures. These normalized OTU tables were used for compositional and comparative 
163 analyses. Column graphs of the ten most abundant phylotypes from each dataset were made

164 for compositional analyses. OTU tables were processed and visualised in R 4.0.3 (19) using

165 phyloseq 1.34.0 (20) and ggplot2 (21).

166 Prior to co-occurrence analysis for network visualisation, OTUs present in less than $10 \%$ of

167 each culture group, $50 \%$ of the lake water samples, or with a relative abundance always

168 below $1 \%$ were removed. This was done to reduce sparcity to below $50 \%$. A network

169 ensemble approach was used where any correlation (edge) between OTUs (node) that was

170 found in at least two of the three following methods was included in the final visualisation:

171 SPIEC-EASI (22), SparcCC (23), and Pearsons correlation. All co-occurrence methods were

172 implemented in R 4.0.3 using spiec-easi 1.1.1 for SPIEC-EASI, SparcCC and the psych 2.1.3

173 package for Pearsons correlation. Networks were visualised in cytoscape.

174 To identify communities that had unbalanced methane cycling (flux), all cultures that had a

175 recorded change in methane concentration and all cultures that had at least one phylotype

176 associated with methane generation or consumption were retained and analysed for OTU co-

177 occurrence.

178

179 Data availability and source code

180 All scripts used to calculate network correlations are available at:

181 https://github.com/rmondav/methane_cycling_networks, v1.0.0 recorded at 182 https://doi.org/10.5281/zenodo.5531947. Time-depth-series amplicons are available under 183 BioProject PRJEB27633 (12). Mixed culture sequence data has been deposited at the 184 European Nucleotide Archive (ENA) at EMBL-EBI under accession number BioProject 185 PRJEB48661 (https://www.ebi.ac.uk/ena/browser/view/PRJEB48661). 186

187 Results 
188 Change in dominant lake community with depth

189 The most abundant OTUs in the lake time-depth-series belonged to just three phyla:

190 Bacteroidetes, Chloroflexi, and Proteobacteria. The surface lake microbial community was

191 dominated by the $\square$-proteobacterial Rhodoferax OTU25 and Polaromonas OTU11 and

192 Methylomonaceae OTU24 while the deeper lake community was increasingly dominated by

193 a Chlorobium OTU9 and Oscillochlorus OTU21 (Fig. 1C). Polynucleobacter OTU2 was

194 highly abundant throughout the lake water (Fig 1C) showing no preference for depth. It was

195 also abundant in most of the mixed cultures (Fig 1D) and was present at low abundance in 196 media controls from Sept 2017 (0.2 $\mu \mathrm{m}$ filterable microbes).

198 Communities in lakes associated with methane cycling

199 Known methanotrophs (obligate methane consumers) in the lake time-depth-series network 200 were associated with either a phototrophic cluster of bacteria (green circle top left) or 201 clustered around a methanogen (gold circle top centre, Figure 2). Carbon fixing phototrophs 202 as detected in this cluster, methanotrophs, and the methanogen are all obligate C1-carbon 203 molecule cyclers. Sulphate-reducing bacteria were also associated with these two clusters.

204 The Oscillochloris OTU21 and Chlorobium OTU9 were dominant phylotypes of deeper lake 205 (green circle Figure 2). The methanotroph Methylomonaceae OTU24 and Polaramonas 206 OTU11 (grey circle bottom Figure 2) were dominant in lake Lomtjärnan surface waters (Fig 207 1B). This cluster (grey circle) was negatively correlated to the other methanotrophs, the 208 phototrophs and the methanogen. Methylotrophs (facultative methane consumers) had no 209 clear association with any of these metabolic clusters.

210

211 Percentage of mixed cultures with growth varied with inoculum source 
212 All inoculated cultures from the 2016 inoculum grew and produced sequences, while $71 \%$ of 213 the cultures from 2017 sampling produced sequences. Despite similar culture conditions,

214 there were distinct differences in the dominant genera in the cultures inoculated with water 215 samples from early spring 2016 compared to autumn 2017 (Fig. 1D).

217 Abundant lineages can both pass through and be retained by $0.22 \mu \mathrm{m}$ filters

218 On average half of the top ten most abundant phylotypes in the time-depth-series and the 2192017 culture-series were also detected in the media control sequenced from 2017. 220 Specifically, five of the most prevalent phylotypes in the 2017 cultures (Pelomonas, 221 Betaproteobacteriales - T34, Polynucleobacter, Sphingomonas, and Staphylococcus) were 222 also detected in the 2017 media control. Seven of the top 25 most abundant OTUs in the 223 time-depth series (Polaromonas, Polynucleobacter, Rhodoferax, Betaproteobacteriales - T34, 224 an unclassified genus of the Sporichthyaceae family, Flavobacterium, and an unclassified 225 genus of Burkholderiaceae) were abundant in media controls.

Detection of methane cycling phylotypes does not always correlate to methane flux measurements

229 The majority (91\%) of cultures had no detectable change in methane concentration or (60\%) 230 had no known methane cycling phylotypes (Figure 3A). Of note in our search for new 231 organisms with a putative role in methane cycling were the 5\% of cultures that had methane 232 flux but no methane cycling phylotypes detected. Also of note were the $36 \%$ of mixed 233 cultures where obligate methane cycling phylotypes were detected but no methane flux was 234 recorded. These two culture groups were noted and selected for further investigation. 
237 Due to the poor correlation between methane flux and methane cycling phylotypes, the co-

238 occurrence patterns of the cultures of note were examined. Organisms detected uniquely in

239 cultures that had unexplained methane concentration were recorded and a putative role in

240 methane cycling proposed (Table 1). Eight microbes associated with the production of

241 methane without detection of archaeal methanogens were noted by " $\mathrm{CH} 4$ production without

242 methanogen". One of these candidates, Rhodopseudomonas, is already annotated as a

243 putative methane producer via the recently documented Fe-only nitrogenase methane release

244 pathway $(24,25)$. Another cohort candidate, Desulfobulbaceae, is a putative sulphate reducer.

245 Many cultures had abundant obligate methane consumers but no change in methane

246 concentration, twenty-five microbes were uniquely associated with these cultures and were

247 noted with "CH4 balance in the presence of methanotroph". There were many Patescibacteria

248 and one putative ammonia oxidizer, Nitrosomonadaceae, in this group. Six bacteria

249 associated with consumption of methane without detection of methane consumers were noted

250 by "CH4 consumption without $\mathrm{CH} 4$ consumer". In total, 39 lineages were identified as

251 potential contributors to methane cycling assemblages.

\section{Discussion}

254 Assembly of microbial communities is cell density and diversity dependent

255 The $71 \%$ to $100 \%$ success rate of culturing in this experiment is in stark contrast to the $10 \%$

256 to $30 \%$ success rate of dilution to extinction where only a few to maybe 20 cells are used in

257 inoculant mixed cultures $(11,26)$. It therefore seems that around 50 cells of a natural

258 community per inoculum (for anaerobic cultures from Lake Lomtjärnan) capture sufficient

259 metabolic breadth to form a functional self-sustaining non-photic community, or cohorts.

260 This for the first time places an upper limit on the minimum metabolic diversity required for

261 functional (anaerobic) aquatic cohorts $(27,28)$. 
263 Filterable microbes play a significant role in natural and laboratory systems

264 A growing literature has discussed and evaluated the aquatic microbial filterable microbes in 265 relation to the concept and efficacy of filter sterilisation of media used for laboratory 266 culturing and environmental role (29-32). Here we also found that some phylotypes e.g., 267 Polynucleobacter, can both pass through filters to be detected in filter 'sterilised' media, and 268 be captured on filters as seen here in the time-depth-series. While the debate over whether 269 filter sterilisation works is mostly complete, a discussion on the effect on abundance 270 estimates of the loss of the filterable microbes might be worth re-opening.

272 Associations between methanotrophs and other lifestyles in the lake

273 The lake time-series network shows an association between photoautotrophic Chlorobium, a 274 methanotrophs, and sulphate reducing bacteria. This connection could be, for example, via 275 carbon fixation by the autotrophic bacteria which also oxidise sulphur species to sulphate 276 which is then consumed by the sulphate reducers. The Methylobacter phylotype, based on 277 associations visualised in the network, is not reliant on proximity to a methanogen to thrive 278 but rather is integral to the phototrophic cohort. The remainder of the methanotrophs in the 279 cluster are associated with a methanogen and sulphate reducers. One of these sulphate 280 reducers, Desulfobulbaceae is a putative methane consumer cohort member, while the other, 281 Geobacter, is a known consort of Methanothrix (synonym Methanosaeta (40)) whereby 282 direct electron transfer allows the methanogen to switch to $\mathrm{CO} 2$ utilisation for methane 283 generation $(44,45)$. Methane is then released into the water and consumed by the cohort of 284 methanotrophs.

285 Except for two cyanobacteria OTUs (Planktothrix and Pseudoanabaeana) most of the taxa in 286 the green and gold circles are associated with oxic-anoxic interfaces. Besides the phototrophs 
287 all of the taxa present in the upper cohort and correlated to methanotrophs are potential 288 microaerophiles e.g. Polyangjaceae or Gallionalaceae (33-35). Also included in the circles 289 are potential anaerobe with known ability to live in microaerophile condition like Geobacter, 290 or Rhodocyclaceae a taxa known to use a wide range of electron acceptor, including but not 291 limited to O2 (36). These correlations between methanotrophs and taxa associated with the 292 oxic anoxic interface is in line with the fact that this interface is a hotspot for methanotrophy 293 (37-39). Based only on correlation it is impossible to tell if the cooccurrence of different 294 OTU is due to shared environmental preference of by necessary interactions. The fact that all 295 the organisms represented in the gold and green circles appear to favorize low oxygen 296 environment suggests that the cooccurrence might be driven by environmental preferences. It 297 is nevertheless interesting to observe that three different OTUs attributed to methanotrophic 298 taxa are directly correlated to a methanogen, Methanothrix (synonym Methanosaeta (40)). 299 The presence of an archaeal methanogen in a potentially oxic environment might seem 300 surprising, but again there is also mounting evidence that methanogenesis isn't limited to 301 anoxic environments $(41,42)$. Furthermore Methanothrix is among the methanogen with a 302 potential to strive in oxic conditions $(42,43)$. Interestingly though the Methanothrix OTU 303 correlates with two OTUs that lean more on the anaerobic side of the interface (Geobacter 304 and Crenothrix).

305 Others interesting correlation are the ones with iron cycling phylotypes (i.e Geobacter and 306 Gallioneallaceae). Indeed both methanogen and methanotrophs have high iron demand for 307 cofactor production (46). Furthermore, it has been suggested that interactions can be 308 favorable to methanotrophs $(47,48)$. But if notable it is, again, impossible to clearly state the 309 nature of the relation between those methane cycling taxa with the iron cycling ones. 
311 All in all, the clusters in the top of the figure suggest that most of the methantrophs detected

312 are attached to an environment with little oxygen favorizing taxa with the ability to deal with

313 change in $\mathrm{O} 2$ concentration through mobility (like Gallionella or Chlorflexi) or metabolic

314 plasticity (e.g Chloroflexi or Rhodocyladeae). Interestingly, the Methylococcales OTU

315 abundances correlates with both anoxic leaning OTU (Chrenothrix, Methanothrix, Geobacter)

316 and an oxygenic phototroph (Planktothrix and Pseudanabaena, both cyanobacteria). This

317 again suggest microoxic condition, and is in line with work suggesting that aerobic

318 methanotrophy can be enhanced by phototroph in low oxygen environment (49).

320 The most probable explanation for the negative correlation between the upper circle clusters

321 (Figure 2) and the grey circle cluster in the bottom of the figure are due to spatial separation

322 likely controlled by temperature. Several OTU present in the grey cluster belongs to taxa

323 including both aerobic and anaerobic species like Polaromonas, Flavobacterium or

324 Burkholdaerieacea. Both Polaromonas and Flavobacterium are generally considered

325 psychrophilic and psychrotolerant taxa respectively $(50,51)$ (52). Furthermore Both

326 Pedobacter and Rhodoferax also include psychrophilic taxa $(53,54)$. The association of these

327 psychrophilic and tolerant taxa with the surface water, might be explained considering that

328 sampling was performed during the ice-covered season it make sense as during winter,

329 stratification is inverted, with the coldest water found at the surface directly beneath ice. It

330 seems therefor very likely that the grey cluster is associated with cold temperature compared

331 to rather than by oxygen concentration.

333 Associations between methanotrophs and other lifestyles in the cohorts in model communities

334 Over the last two decades there has been a large increase in the catalogued number of 335 microbes and metabolic pathways capable of generating, releasing, or consuming methane 
$336(55,56)$. Both increases are likely to continue as we explore deeper genomic and metabolic

337 space. For these reasons it is not a surprise that methane consumption and production did not

338 correlate clearly with detection of known methane cycling phylotypes. It was surprising

339 however, the degree to which these were decoupled. It therefore not clear if this uncoupling is

340 due to poor flux detection, or low abundance of methane cycling organisms. Previously, it

341 has been demonstrated that methanotroph abundance can be uncoupled form methane

342 oxidation rates (58-60).

343

344 The generation here of a list of 39 novel phylotypes that may be directly or indirectly

345 involved in methane cycling is a mere indicator of how much more work can be done to

346 identify key players effecting cycling of methane and other C1 molecules.

347 We hypothesize that the participate in methane cycle is not only related to the presence of 348 organisms with the known ability to produce or use methane, but that their cohorts as found 349 in the model communities might be key controllers in methane cycling.

\section{Acknowledgments}

352 SLG was supported by a SciLifeLab fellowship, a Stipend from King Carl XVI Gustav's

353 science foundation to research methane oxidation in lakes. SP was support by a SciLifeLab

354 fellowship. Field, laboratory, and DNA amplicon sequencing was enabled by grants from

355 Olsson-Borghs Stiftelse.

356 Computations were enabled by resources in projects 2020-5-529 \& 2020-15-261 and data 357 storage projects 2020-6-164 \& 2020-16-196 provided by the Swedish National Infrastructure 358 for Computing (SNIC) at UPPMAX which is partially funded by the Swedish Research

359 Council through grant agreement no. 2018-05973. 
360 SP, GM, SLG conceived of and implemented field and laboratory research. SP and SLG

361 obtained financial and material resources while SP and RM obtained computational

362 resources. RM and SLG wrote and implemented scripts for analysis and visualization of

363 results. RM and SP drafted the manuscript and all authors contributed to discussions, editing,

364 and approval of final manuscript. RM curated data and scripts. SP and SLG supervised and

365 SLG coordinated the project.

367 References

368 1. Forster P, Ramaswamy V, Artaxo P, Berntsen T, Betts R, Fahey DW, Haywood J, 369 Lean J, Lowe DC, Myhre G, Nganga J, Prinn R, Raga G, Schulz M, Dorland R Van. 2007. 370 Changes in Atmospheric Constituents and in Radiative ForcingClimate Change 2007: The 371 Physical Science Basis. Contribution of Working Group I to the Fourth Assessment Report of 372 the Intergovernmental Panel on Climate Change, AR4 ed. Cambridge University Press, 373 Cambridge.

374 2. Fernández-Amador O, Francois JF, Oberdabernig DA, Tomberger P. 2020. The 375 methane footprint of nations: Stylized facts from a global panel dataset. Ecol Econ $376 \quad 170: 106528$.

377 3. Frenzel P, Thebrath B, Conrad R. 1990. Oxidation of methane in the oxic surface 378 layer of a deep lake sediment (Lake Constance). FEMS Microbiol Ecol 73:149-158.

379 4. Kankaala P, Huotari J, Peltomaa E, Saloranta T, Ojala A. 2006. Methanotrophic 380 activity in relation to methane efflux and total heterotrophic bacterial production in a 381 stratified, humic, boreal lakeLimnol. Oceanogr.

382 5. Bastviken D, Tranvik LJ, Downing JA, Crill PM, Enrich-prast A. 2011. the 383 Continental Carbon Sink. Science (80- ) 331:6. 
384 6. Mayr M, Zimmermann M, Dey J, Wehrli B, Bürgmann H. 2020. Lake mixing regime

385 selects methane-oxidation kinetics of the methanotroph assemblage. Biogeosciences Discuss $386 \quad 1-15$.

387 7. Sawakuchi HO, Martin G, Peura S, Bertilsson S, Karlsson J, Bastviken D. 2021. 388 Phosphorus Regulation of Methane Oxidation in Water From Ice $\square$ Covered Lakes. J Geophys 389 Res Biogeosciences 126:e2020JG006190.

390 8. Oswald K, Milucka J, Brand A, Littmann S, Wehrli B, Kuypers MMM, Schubert CJ. 391 2015. Light-dependent aerobic methane oxidation reduces methane emissions from 392 seasonally stratified lakes. PLoS One 10:e0132574.

393 9. Garcia S. 2016. Mixed cultures as model communities: hunting for ubiquitous 394 microorganisms, their partners, and interactions. Aquat Microb Ecol 77:79-85.

395 10. Garcia SL, Buck M, McMahon KD, Grossart H-P, Eiler A, Warnecke F. 2015. 396 Auxotrophy and intrapopulation complementary in the 'interactome' of a cultivated 397 freshwater model community. Mol Ecol 24:4449-4459.

398 11. Mondav R, Bertilsson S, Buck M, Langenheder S, Lindström ES, Garcia SL. 2020.

399 Streamlined and abundant bacterioplankton thrive in functional cohorts. mSystems 5:e0031640020.

401 12. Garcia SL, Szekely AJ, Bergvall C, Schattenhofer M, Peura S. 2019. Decreased Snow 402 Cover Stimulates Under-Ice Primary Producers but Impairs Methanotrophic Capacity. 403 mSphere 4.

404 13. Herlemann DP, Labrenz M, Jürgens K, Bertilsson S, Waniek JJ, Andersson AF. 2011. 405 Transitions in bacterial communities along the $2000 \square \mathrm{km}$ salinity gradient of the Baltic Sea. 406 ISME J 5:1571-9.

407 14. Kozich JJ, Westcott SL, Baxter NT, Highlander SK, Schloss PD. 2013. Development 408 of a Dual-Index Sequencing Strategy and Curation Pipeline for Analyzing Amplicon 
409 Sequence Data on the MiSeq Illumina Sequencing Platform. Appl Environ Microbiol $410 \quad 79: 5112-5120$.

411 15. Rognes T, Flouri T, Nichols B, Quince C, Mahé F. 2016. VSEARCH: a versatile open 412 source tool for metagenomics. PeerJ 4:e2584.

413 16. Schloss PD, Westcott SL, Ryabin T, Hall JR, Hartmann M, Hollister EB, Lesniewski 414 RA, Oakley BB, Parks DH, Robinson CJ, Sahl JW, Stres B, Thallinger GG, Van Horn DJ, 415 Weber CF. 2009. Introducing mothur: Open-source, platform-independent, community416 supported software for describing and comparing microbial communities. Appl Environ 417 Microbiol 75:7537-7541.

418 17. Quast C, Pruesse E, Yilmaz P, Gerken J, Schweer T, Yarza P, Peplies J, Glöckner FO. 419 2013. The SILVA ribosomal RNA gene database project: improved data processing and web420 based tools. Nucleic Acids Res 41:D590-6.

421 18. Caporaso JG, Kuczynski J, Stombaugh J, Bittinger K, Bushman FD, Costello EK, 422 Fierer N, Peña AG, Goodrich JK, Gordon JI, Huttley GA, Kelley ST, Knights D, Koenig JE, 423 Ley RE, Lozupone CA, McDonald D, Muegge BD, Pirrung M, Reeder J, Sevinsky JR, 424 Turnbaugh PJ, Walters WA, Widmann J, Yatsunenko T, Zaneveld J, Knight R. 2010. QIIME 425 allows analysis of high- throughput community sequencing data. Nat Methods 7:335-336.

426 19. R Core Development Team. 2015. R: a language and environment for statistical 427 computing, 3.2.1. Doc Free available internet http//www r-project org.

428 20. McMurdie PJ, Holmes S. 2013. phyloseq: An R Package for Reproducible Interactive 429 Analysis and Graphics of Microbiome Census Data. PLoS One 8:e61217.

430 21. Ginestet C. 2011. ggplot2: Elegant Graphics for Data Analysis. J R Stat Soc Ser A 431 (Statistics Soc 174:245-246. 
432 22. Kurtz ZD, Müller CL, Miraldi ER, Littman DR, Blaser MJ, Bonneau RA. 2015.

433 Sparse and Compositionally Robust Inference of Microbial Ecological Networks. PLOS

434 Comput Biol 11:e1004226.

435 23. Friedman J, Alm EJ. 2012. Inferring Correlation Networks from Genomic Survey 436 Data. PLoS Comput Biol 8:e1002687.

437 24. Fixen KR, Zheng Y, Harris DF, Shaw S, Yang Z-Y, Dean DR, Seefeldt LC, Harwood 438 CS. 2016. Light-driven carbon dioxide reduction to methane by nitrogenase in a 439 photosynthetic bacterium. Proc Natl Acad Sci 113:10163-10167.

440 25. McRose DL, Zhang X, Kraepiel AML, Morel FMM. 2017. Diversity and activity of 441 alternative nitrogenases in sequenced genomes and coastal environments. Front Microbiol.

442 26. Henson MW, Lanclos VC, Pitre DM, Weckhorst JL, Lucchesi AM, Cheng C, 443 Temperton B, Thrash JC. 2020. Expanding the diversity of bacterioplankton isolates and 444 modeling isolation efficacy with large-scale dilution-to-extinction cultivation. Appl Environ 445 Microbiol 86.

446 27. Yu X, Polz MF, Alm EJ. 2019. Interactions in self-assembled microbial communities 447 saturate with diversity. ISME J 13:1602-1617.

448 28. Pascual-García A, Bonhoeffer S, Bell T. 2020. Metabolically cohesive microbial 449 consortia and ecosystem functioning. Philos Trans R Soc Lond B Biol Sci 375:20190245.

450 29. Hahn MW. 2004. Broad diversity of viable bacteria in "sterile" $(0.2 \mu \mathrm{m})$ filtered 451 water. Res Microbiol 155:688-691.

452 30. Nakai R. 2020. Size matters: Ultra-small and filterable microorganisms in the 453 environment. Microbes Environ.

454 31. Ghuneim LAJ, Distaso MA, Chernikova TN, Bargiela R, Lunev EA, Korzhenkov 455 AA, Toshchakov S V., Rojo D, Barbas C, Ferrer M, Golyshina O V., Golyshin PN, Jones 
456 DL. 2021. Utilization of low-molecular-weight organic compounds by the filterable fraction 457 of a lotic microbiome. FEMS Microbiol Ecol 97:1-16.

458 32. Maejima Y, Kushimoto K, Muraguchi Y, Fukuda K, Miura T, Yamazoe A, Kimbara

459 K, Shintani M. 2018. Proteobacteria and Bacteroidetes are major phyla of filterable bacteria 460 passing through $0.22 \mu \mathrm{m}$ pore size membrane filter, in Lake Sanaru, Hamamatsu, Japan. 461 Biosci Biotechnol Biochem 82:1260-1263.

462 33. Pedersen K. 2011. Gallionella, p. 411-412. In Reitner, J, Thiel, V (eds.), 463 Encyclopedia of Geobiology. Springer Netherlands, Dordrecht.

464 34. Hanert HH. 2006. The Genus Gallionella, p. 990-995. In Dworkin, M, Falkow, S, 465 Rosenberg, E, Schleifer, K-H, Stackebrandt, E (eds.), The Prokaryotes: Volume 7: 466 Proteobacteria: Delta, Epsilon Subclass. Springer, New York, NY.

467 35. Garcia R, Müller R. 2014. The Family Polyangiaceae, p. 247-279. In Rosenberg, E, 468 DeLong, EF, Lory, S, Stackebrandt, E, Thompson, F (eds.), The Prokaryotes: 469 Deltaproteobacteria and Epsilonproteobacteria. Springer, Berlin, Heidelberg.

470 36. Oren A. 2014. The Family Methanoregulaceae, p. 253-258. In Rosenberg, E, 471 DeLong, EF, Lory, S, Stackebrandt, E, Thompson, F (eds.), The Prokaryotes: Other Major 472 Lineages of Bacteria and The Archaea. Springer, Berlin, Heidelberg.

473 37. Rissanen AJ, Saarenheimo J, Tiirola M, Peura S, Aalto SL, Karvinen A, Nykanen H. 474 2018. Gammaproteobacterial methanotrophs dominate methanotrophy in aerobic and 475 anaerobic layers of boreal lake waters. Aquat Microb Ecol 81:257-276.

476 38. Reis PCJ, Thottathil SD, Ruiz $\square$ González C, Prairie YT. 2020. Niche separation 477 within aerobic methanotrophic bacteria across lakes and its link to methane oxidation rates. 478 Environ Microbiol 22:738-751.

479 39. Samad MS, Bertilsson S. 2017. Seasonal Variation in Abundance and Diversity of 480 Bacterial Methanotrophs in Five Temperate Lakes. Front Microbiol 8. 
481 40. Boone DR. 1991. Strain GP6 is proposed as the neotype strain of Methanothrix 482 soehngenii(VP) pro synon. Methanothrix concilii(VP) and Methanosaeta concilii(VP):

483 Request for an opinion. Int J Syst Bacteriol 41:588-589.

484 41. Bogard MJ, del Giorgio PA, Boutet L, Chaves MCG, Prairie YT, Merante A, Derry 485 AM. 2014. Oxic water column methanogenesis as a major component of aquatic CH4 fluxes. 486 Nat Commun 5:5350.

487 42. Angle JC, Morin TH, Solden LM, Narrowe AB, Smith GJ, Borton MA, Rey-Sanchez 488 C, Daly RA, Mirfenderesgi G, Hoyt DW, Riley WJ, Miller CS, Bohrer G, Wrighton KC. 489 2017. Methanogenesis in oxygenated soils is a substantial fraction of wetland methane 490 emissions. Nat Commun 8:1567.

491 43. Jetten MS, Stams AJ, Zehnder AJ. 1989. Purification and characterization of an 492 oxygen-stable carbon monoxide dehydrogenase of Methanothrix soehngenii. Eur J Biochem $493 \quad 181: 437-441$.

494 44. Holmes DE, Shrestha PM, Walker DJF, Dang Y, Nevin KP, Woodard TL, Lovley 495 DR. 2017. Metatranscriptomic evidence for direct interspecies electron transfer between 496 Geobacter and Methanothrix species in methanogenic rice paddy soils. Appl Environ 497 Microbiol 83.

498 45. Liu C, Sun D, Zhao Z, Dang Y, Holmes DE. 2019. Methanothrix enhances biogas 499 upgrading in microbial electrolysis cell via direct electron transfer. Bioresour Technol $500 \quad 291: 121877$.

501 46. Johnson C, England A, Munro-Ehrlich M, Colman DR, DuBois JL, Boyd ES. 2021. 502 Pathways of Iron and Sulfur Acquisition, Cofactor Assembly, Destination, and Storage in 503 Diverse Archaeal Methanogens and Alkanotrophs. J Bacteriol 203:e0011721. 
504 47. Ho A, de Roy K, Thas O, De Neve J, Hoefman S, Vandamme P, Heylen K, Boon N.

505 2014. The more, the merrier: heterotroph richness stimulates methanotrophic activity. ISME J

$506 \quad 8: 1945-1948$.

507 48. Iguchi H, Yurimoto H, Sakai Y. 2011. Stimulation of Methanotrophic Growth in 508 Cocultures by Cobalamin Excreted by Rhizobia. Appl Environ Microbiol 77:8509-8515.

509 49. van Grinsven S, Oswald K, Wehrli B, Jegge C, Zopfi J, Lehmann MF, Schubert CJ.

510 2021. Methane oxidation in the waters of a humic-rich boreal lake stimulated by 511 photosynthesis, nitrite, Fe(III) and humics. Biogeosciences 18:3087-3101.

512 50. Darcy JL, Lynch RC, King AJ, Robeson MS, Schmidt SK. 2011. Global Distribution 513 of Polaromonas Phylotypes - Evidence for a Highly Successful Dispersal Capacity. PLoS 514 One 6:e23742.

515 51. Michaud L, Caruso C, Mangano S, Interdonato F, Bruni V, Lo Giudice A. 2012. 516 Predominance of Flavobacterium, Pseudomonas, and Polaromonas within the prokaryotic 517 community of freshwater shallow lakes in the northern Victoria Land, East Antarctica. FEMS 518 Microbiol Ecol 82:391-404.

519 52. Bernardet J-F, Bowman JP. 2006. The Genus Flavobacterium, p. 481-531. In 520 Dworkin, M, Falkow, S, Rosenberg, E, Schleifer, K-H, Stackebrandt, E (eds.), The 521 Prokaryotes: Volume 7: Proteobacteria: Delta, Epsilon Subclass. Springer, New York, NY.

522 53. Viana AT, Caetano T, Covas C, Santos T, Mendo S. 2018. Environmental superbugs:

523 The case study of Pedobacter spp. Environ Pollut 241:1048-1055.

524 54. Finneran KT, Johnsen C V., Lovley DRY 2003. Rhodoferax ferrireducens sp. nov., a 525 psychrotolerant, facultatively anaerobic bacterium that oxidizes acetate with the reduction of 526 Fe(III). Int J Syst Evol Microbiol 53:669-673.

527 55. Kurth JM, Op den Camp HJM, Welte CU. 2020. Several ways one goal528 methanogenesis from unconventional substrates. Appl Microbiol Biotechnol 104:6839-6854. 
529 56. Berghuis BA, Yu FB, Schulz F, Blainey PC, Woyke T, Quake SR. 2019.

530 Hydrogenotrophic methanogenesis in archaeal phylum Verstraetearchaeota reveals the shared

531 ancestry of all methanogens. Proc Natl Acad Sci U S A 116:5037-5044.

532 58. DeBruyn JM, Leigh-Bell JA, McKay RML, Bourbonniere RA, Wilhelm SW. 2004.

533 Microbial Distributions and the Impact of Phosphorus on Bacterial Activity in Lake Erie. J

534 Great Lakes Res 30:166-183.

535 59. Vrede K, Vrede T, Isaksson A, Karlsson A. 1999. Effects of nutrients (phosphorous, 536 nitrogen, and carbon) and zooplankton on bacterioplankton and phytoplankton-a seasonal 537 study. Limnol Oceanogr 44:1616-1624.

538 60. Zheng Y, Zhang L, He J-Z. 2013. Immediate effects of nitrogen, phosphorus, and 539 potassium amendments on the methanotrophic activity and abundance in a Chinese paddy 540 soil under short-term incubation experiment. J Soils Sediments 13:189-196. 
bioRxiv preprint doi: https://doi. org/10.1101/2022.01 24.477581 t this version posted January 25, 2022 . The copyright holder for this preprint (which was not certified by peer review) is the author/funder, who has granted bioRxiv a license to display the preprint in perpetuity. It is made available under aCC-BY 4.0 International license.

A
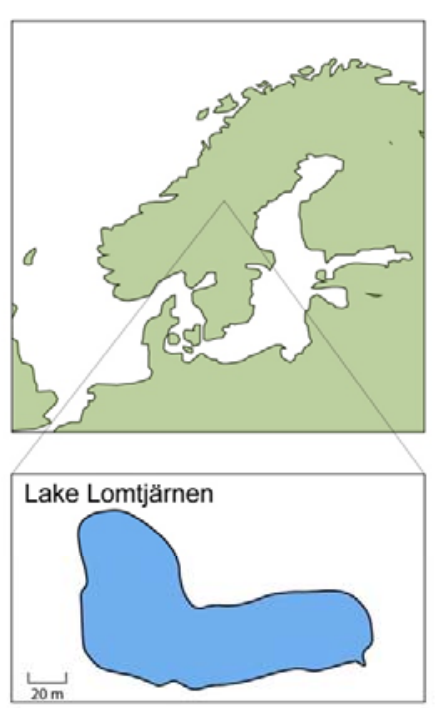

B

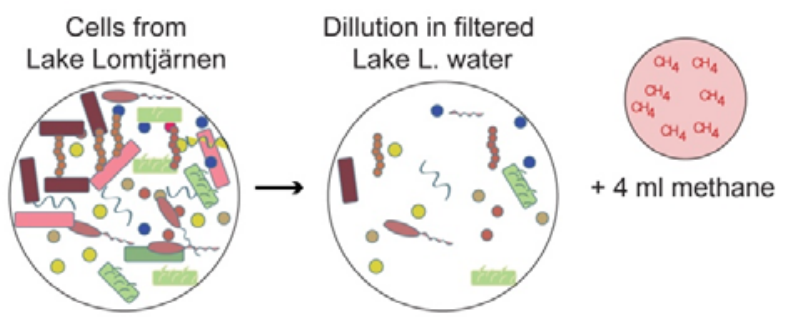

10 ml Lake Lomtjärnen culture x 166 replicates

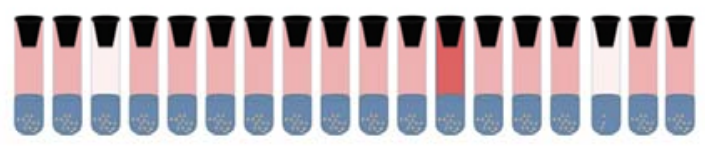

2 month incubation

C

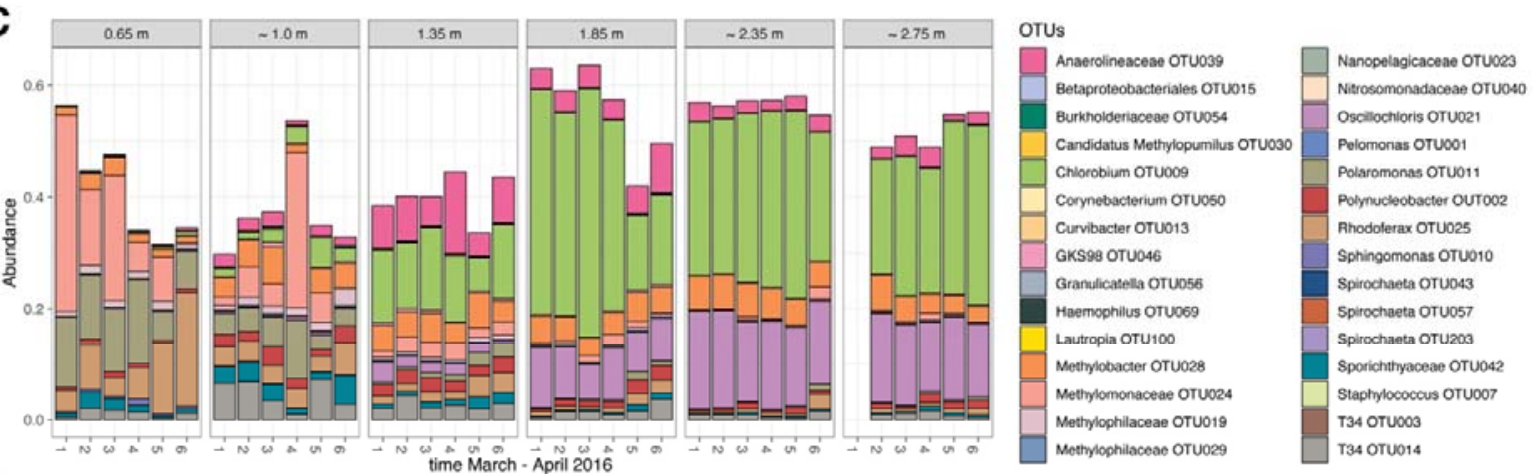

D

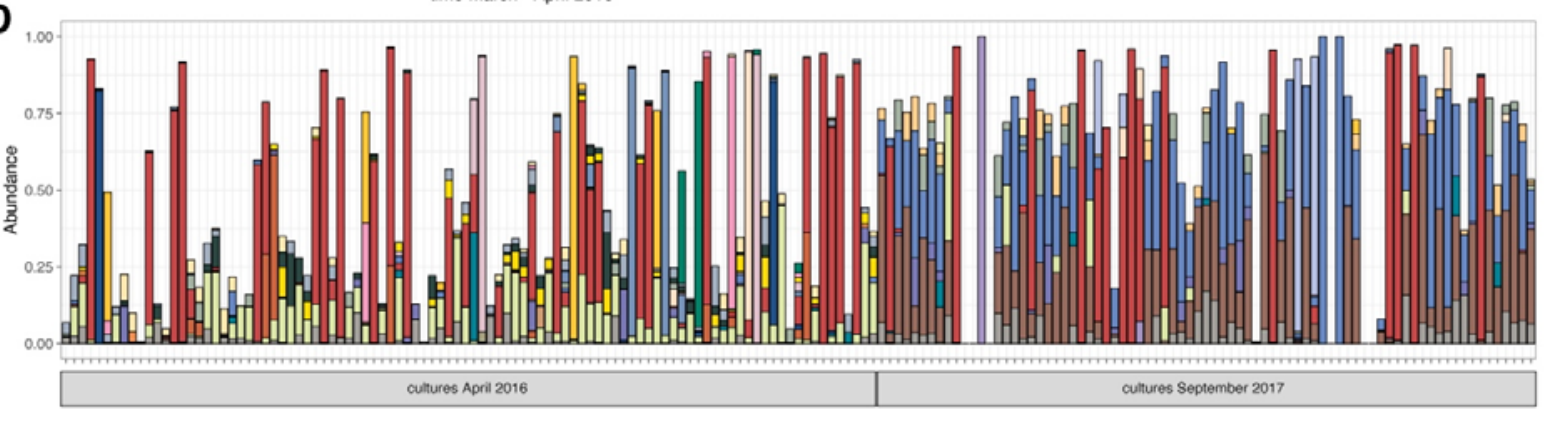

546 Figure 1. Sample location [A], schematic of dilution and set up of model communities [B],

547 most abundant OTUs detected in lake timeseries [C], and most abundant OTUs from model

548 communities [D]. The legend in $\mathrm{C}$ is also for $\mathrm{D}$. 


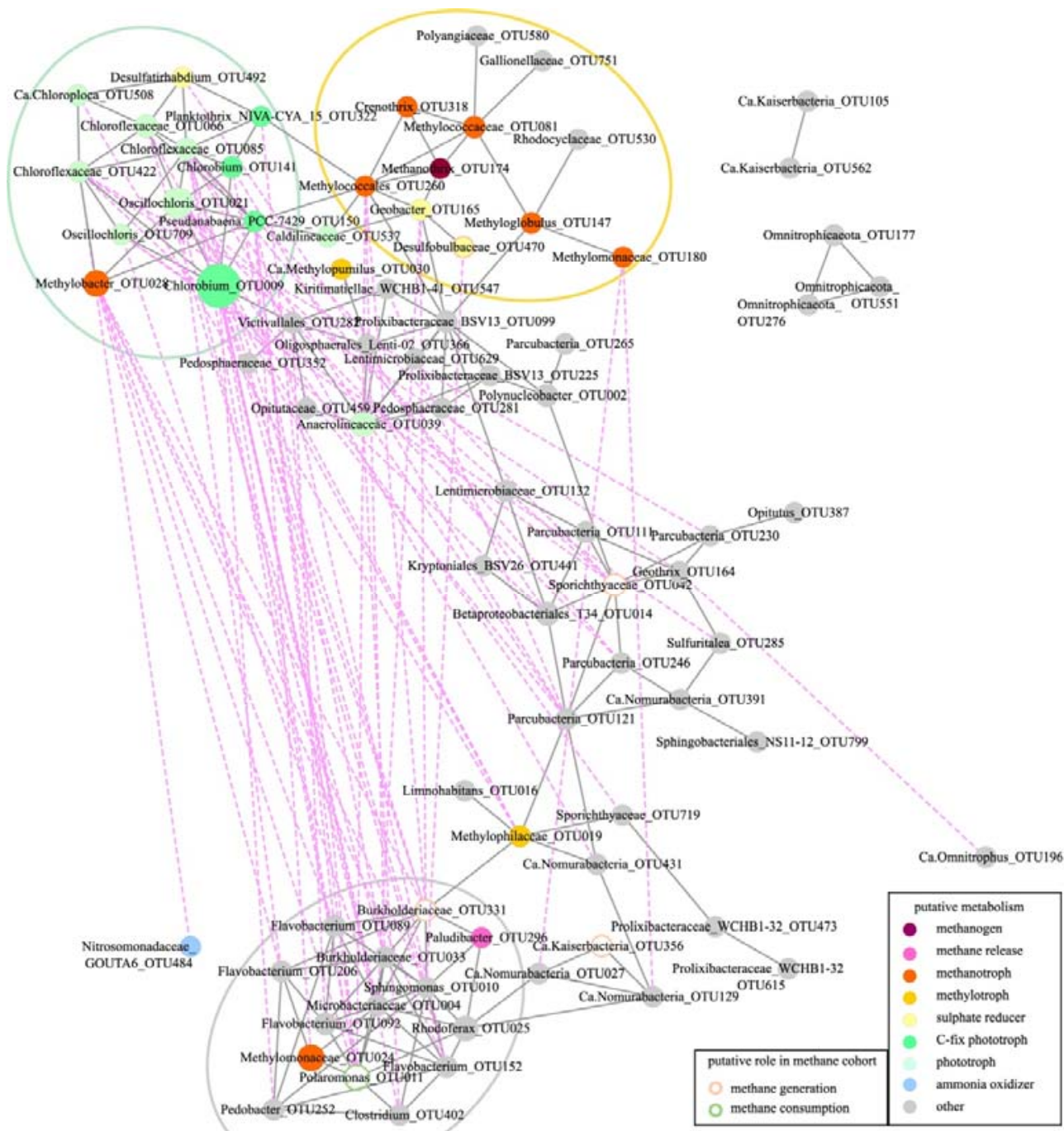

551 Figure 2. Lake time-depth-series network. Filled node-circles are OTUs with the following

552 color codes: methanogens-red, methanotrophs-orange, methylotrophs-golden, C-fixing

553 phototrophs-bright green, other phototrophs-pale green, sulphate reducers-yellow. Grey lines

554 show positive correlations and pink-dotted lines show negative correlations. Green, golden,

555 and grey bigger circles designate cohort clusters. We designated putative roles in methane

556 consumption from observations in the dilution model communities and they are shown in not-

557 filled node-circles in peach and green. 


\section{A All cultures in the study}

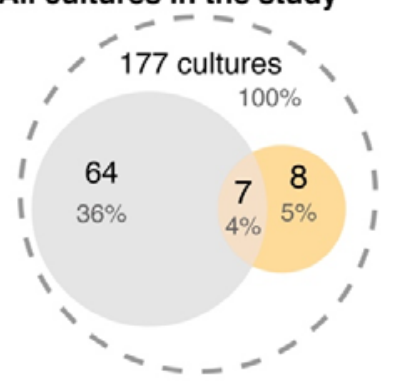

C Cultures from September 2017

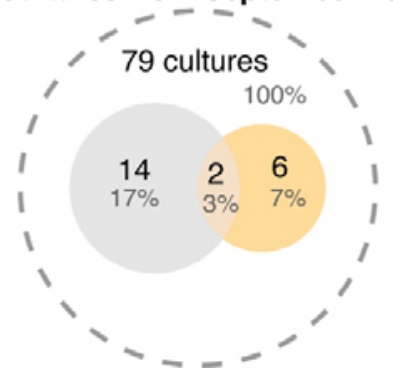

\section{B Cultures from April 2016}

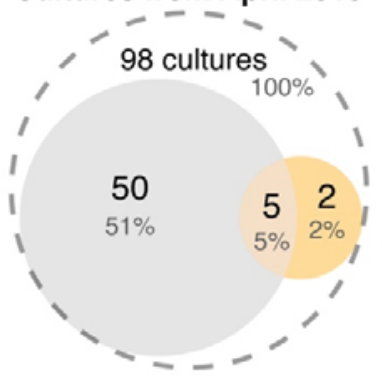

560

561

562 Figure 3. Venn diagram of all cultures in this study [A], cultures from April 2016 [B] and

563 cultures from September 2017 [C]. In orange the cultures with measured methane flux and in

564 grey the cultures with detection of (known) methane cycling phylotypes.

565

566 
bioRxiv preprint doi: https://doi.org/10.1101/2022.01.24.477581; this version posted January 25, 2022. The copyright holder for this preprint (which was not certified by peer review) is the author/funder, who has granted bioRxiv a license to display the preprint in perpetuity. It is made available under aCC-BY 4.0 International license.

\section{Table 1. Phylotypes in this study with a potential role in methane cycling.}

\section{phylum \\ Patescibacteria

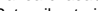

Patescibacteria

Proteobacteria

Patescibacteria

Proteobacteria

Proteobacteria

Actinobacteria

Actinobacteria
Actinobacteria

Actinobacteria

Bacteroidetes

Firmicutes

Nanoarchaeaeota

Patescibacteria

Patescibacteria

Patescibacteria

Patescibacteria

Patescibacteria

Patescibacteria

Patescibacteria

Patescibacteria

Patescibacteria

Patescibacteria

Patescibacteria
Planctomycetes

Proteobacteria

Proteobacteria

Proteobacteria

Proteobacteria

Proteobacteria

Bacteroidetes

Proteobacteria

Firmicutes

Proteobacteria

Actinobacteria
Proteobacteria

\section{Ca.Vogelbacteria_fa \\ Woesearchaeia_fa}

Ca.Staskawiczbacteria fa

Burkholderiaceae

ubacteria_fa

Desulfobulbaceae

Xanthobacteraceae

Korynebacteriacea

Minerococracaceae

Streptomycetaceae

SB-5

Erysipelotrichaceae

Woesearchaeia_fa

Ca.Adlerbacteria_fa

Ca.Azambacteria_fa

Ca.Kaiserbacteria_fa

Ca.Moranbacteria_fa

Ca.Nomurabacteria_fa

Ca.Staskawiczbacteria

Ca.Woesebacteria_fa

Ca.Yano

Parcubacteria

WWE3_fa

Pirellulaceae

Burkholderiaceae

Coxiellaceae

Hyphomonadaceae
Nitrosomonadaceae

Weeksellaceae

Burkholderiaceae

Clostridiaceae_1

Xanthomonadaceae

Corynebacteriacea
Beijerinckiaceae genus

Ca.Vogelbacteria_ge

a.Staskawiczbacteria_

Burkholderiaceae_ge

Parcubacteria_ge

Rhodopseudomonas

Corynebacterium_1

Kineosporiaceae_ge

Rothia

Streptomyces

SB-5_ge

Solobacterium

Woesearchaeia_ge

Ca.Adlerbacteria_ge

Ca.Azambacteria_ge

Ca.Kaiserbacteria_ge

a.Moranbacteria_ge

Ca.Nomurabacteria_ge

Ca.Woesebacteria_ge

ge unknown
ia_ge unknown

CPR2_ge

Parcubacteria_s

WWE3_ge

uncultured

Acidovorax
Ralstonia

Coxiella

Hirschia

uncultured

Cloacibacterium

Tepidimonas

Fonticella

Pseudoxanthomonas

Lawsonella

Beijerinckiaceae_ge metabolism culture note

unknown

.

unknown

SO4_redctn

put $\mathrm{N} 2, \mathrm{CH} 4$

unknown

unknown

unknown

unknown
unknown

unknown

unknown

unknown

unknown

unknown

unknown

unknown

unknown

unknown

unknown

unknown

NH3 oxidizer

unknown

unknown

unknown

unknown

unknown
$\mathrm{CH} 4$ production without methanogen $\mathrm{CH} 4$ production without methanogen $\mathrm{CH} 4$ production without methanogen $\mathrm{CH} 4$ production without methanogen CH4 production without methanogen CH4 production without methanogen

CH4 production without methanogen

$\mathrm{CH} 4$ balance in the presence of methantroph $\mathrm{CH} 4$ balance in the presence of methantroph $\mathrm{CH} 4$ balance in the presence of methantrop $\mathrm{CH} 4$ balance in the presence of methantroph $\mathrm{CH} 4$ balance in the presence of methantroph $\mathrm{CH} 4$ balance in the presence of methantroph $\mathrm{CH} 4$ balance in the presence of methantroph $\mathrm{CH} 4$ balance in the presence of methantrop $\mathrm{CH} 4$ balance in the presence of methantrop $\mathrm{CH} 4$ balance in the presence of methantroph $\mathrm{CH} 4$ balance in the presence of methantroph $\mathrm{CH} 4$ balance in the presence of methantroph $\mathrm{CH} 4$ balance in the presence of methantroph $\mathrm{CH} 4$ balance in the presence of methantroph $\mathrm{CH} 4$ balance in the presence of methantrop CH4 balance in the presence of methantroph CH4 balance in the presence of methantroph $\mathrm{CH} 4$ bala in in the presence of methantroph $\mathrm{CH} 4$ balance in the presence of methantroph $\mathrm{CH} 4$ balance in the presence of methantroph $\mathrm{CH} 4$ balance in the presence of methantrop $\mathrm{CH} 4$ balance in the presence of methantroph $\mathrm{CH} 4$ balance in the presence of methantroph $\mathrm{CH} 4$ consumption without $\mathrm{CH} 4$ consumer $\mathrm{CH} 4$ consumption without $\mathrm{CH} 4$ consumer $\mathrm{CH} 4$ consumption without $\mathrm{CH} 4$ consume $\mathrm{CH} 4$ consumption without $\mathrm{CH} 4$ consumer $\mathrm{CH} 4$ consumption without $\mathrm{CH} 4$ consume $\mathrm{CH} 4$ consumption without $\mathrm{CH} 4$ consume putative role in $\mathrm{CH} 4$ cycling $\mathrm{CH} 4$ producer or their cohor $\mathrm{CH} 4$ producer or their cohor $\mathrm{CH} 4$ producer or their cohor $\mathrm{CH} 4$ producer or their cohon $\mathrm{CH} 4$ produeer or their methanogen cohort methanogen cohort
$\mathrm{CH} 4$ producer

$\mathrm{CH} 4$ producer $\mathrm{CH} 4$ producing cohort or methanotroph inhibitor $\mathrm{CH} 4$ producing cohort or methanotroph inhibitor $\mathrm{CH} 4$ producing cohort or methanotroph inhibitor $\mathrm{CH} 4$ producing cohort or methanotroph inhibitor $\mathrm{CH} 4$ producing cohort or methanotroph inhibitor $\mathrm{CH} 4$ producing cohort or methanotroph inhibitor $\mathrm{CH} 4$ producing cohort or methanotroph inhibitor $\mathrm{CH} 4$ producing cohort or methanotroph inhibitor $\mathrm{CH} 4$ producing cohort or methanotroph inhibitor CH4 producing cohort or methanotroph inhibitor CH4 producing cohort or methanotroph inhibitor CH4 producing cohort or methanotroph inhibitor CH4 producing cohort or methanotroph inhibitor CH4 producing cohort or methanotroph inhibitor $\mathrm{CH} 4$ produng cohort or methanotroph inhibitor CH4 producing cont or mer $\mathrm{CH} 4$ producing cohort or methan $\mathrm{CH} 4$ producing cohort or methanotroph inhibitor $\mathrm{CH} 4$ producing cohort or methanotroph inhibitor $\mathrm{CH} 4$ producing cohort or methanotroph inhibitor $\mathrm{CH} 4$ producing cohort or methanotroph inhibitor $\mathrm{CH} 4$ producing cohort or methanotroph inhibitor $\mathrm{CH} 4$ producing cohort or methanotroph inhibitor $\mathrm{CH} 4$ consumer or their cohort

$\mathrm{CH} 4$ consumer or their cohort $\mathrm{CH} 4$ consumer or their cohor $\mathrm{CH} 4$ consumer or their cohor $\mathrm{CH} 44$ consumer or their cohort
$\mathrm{CH} 4$ consumer or their cohort 